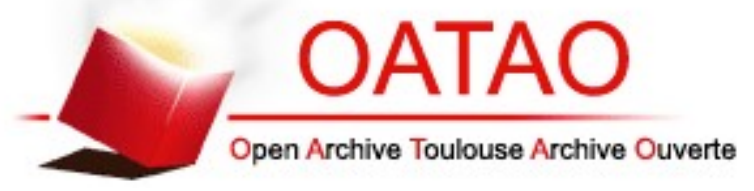

Open Archive Toulouse Archive Ouverte

OATAO is an open access repository that collects the work of Toulouse researchers and makes it freely available over the web where possible

This is an author's version published in:

http://oatao.univ-toulouse.fr/26249

To cite this version: Shen, Hong and Barthélémy, Chloé and Khoury, Elise and Zemmoura, Ilyess and Reménieras, Jean-Pierre and Basarab, Adrian and Kouamé, Denis High-resolution and high-sensitivity blood flow estimation using deconvolution and optimization approaches with application to thyroid vascularization imaging. (2019) In: IEEE International Ultrasonics Symposium (IUS 2019), 6 October 2019 - 9 October 2019 (Glasgow, United Kingdom).

Any correspondence concerning this service should be sent to the repository administrator: tech-oatao@listes-diff.inp-toulouse.fr 


\title{
High-resolution and high-sensitivity blood flow estimation using optimization approaches with application to vascularization imaging
}

\author{
H. Shen ${ }^{1}$, C. Barthelemy ${ }^{2}$, E. Khoury ${ }^{2}$, Y. Zemmoura ${ }^{2}$, JP. Remeniéras ${ }^{2}$, A. Basarab ${ }^{1}$, and D. Kouamé ${ }^{1}$ \\ ${ }^{1}$ IRIT, Université de Toulouse, France. \\ ${ }^{2}$ UMR 1253, iBrain, Université de Tours, Inserm, Tours, France.
}

\begin{abstract}
In this paper, we address the problem of highresolution flow estimation in medical ultrasound images. Imaging methods based on ultrafast sequences associated with adaptive spatiotemporal SVD clutter filtering have recently improved blood flow detection. Herein, we investigate a new way of addressing the clutter filtering problem in order to obtain a highresolution flow estimation, through solving an inverse problem corresponding to both deconvolution and robust principal component analysis. Applied to tissue vascularization imaging via power Doppler images, the proposed method highlights finer details on experimental data compared to existing approaches.

Index Terms-Blood flow, medical ultrasound, sensitive Doppler,robust PCA, deconvolution, optimization.
\end{abstract}

\section{INTRODUCTION}

Recently, blood flow detection in medical ultrasound imaging has been greatly improved by using ultrafast sequences associated with adaptive spatiotemporal singular values decomposition (SVD) clutter filtering [1]. Through this decomposition, the filtering strategy is based on empirical thresholding the correlation of spatial singular vectors magnitude. This leads to the separation of the subspaces corresponding to respectively the tissues and the blood flow [2]. The empirical choice of the optimal threshold is however not obvious in most of practical applications. An alternative solution consists in using robust principal component analysis (RPCA) techniques [3]-[6]. In order to improve these techniques, different attempts have been done in ultrasound imaging, either using sparse priors in a specific basis [7], or sparse coding through a specific dictionary [8]. However, none of them investigated the inherent low resolution of ultrasound Doppler data related to the system point spread function (PSF).

In this paper we investigate a new way of separating blood flow and tissues by accounting for the system PSF. More precisely, we solved an inverse problem related to both deconvolution and RPCA in order to obtain a high-resolution and high-sensitivity blood flow estimation.

\section{MODEL STATEMENT AND RELATED WORKS}

We denote by $S \in \mathbb{C}^{N_{z} N_{x} \times N_{t}}$ the Casorati matrix obtained from 3D IQ complex (number) Doppler data, recorded via ultrafast imaging, with depth $N_{z}$, probe width $N_{x}$ and acquisition time $N_{t}$. The most common method used to filter the clutter and recover the blood flow consists in first performing singular value decomposition (SVD) of $\boldsymbol{S}$ and second setting two thresholds to identify the subspaces of blood, tissue (clutter) and noise, based on the corresponding singular values and vectors.

Alternatively, $\boldsymbol{S}$ can be modelled as:

$$
S=T+B+N
$$

with $\boldsymbol{T} \in \mathbb{C}^{N_{z} N_{x} \times N_{t}}$ the tissue, $\boldsymbol{B} \in \mathbb{C}^{N_{z} N_{x} \times N_{t}}$ the blood, and $\boldsymbol{N} \in \mathbb{C}^{N_{z} N_{x} \times N_{t}}$ the noise matrices. Assuming that the blood (i.e., the flow) is sparse and the tissue weakly changes or moves over time, reasonable assumptions in most of practical applications, it is thus possible to estimate $\boldsymbol{B}$ from $\boldsymbol{S}$. Classically, sparsity is catched in a tractable manner by the $l_{1}$-norm and weak changes or high correlation by the nuclear norm $\|.\|_{*}$. This results in solving an inverse problem in which the estimation of $\boldsymbol{B}$ and $\boldsymbol{T}$, say $(\hat{\boldsymbol{B}}, \hat{\boldsymbol{T}})$, can be obtained by minimizing the following function:

$$
(\hat{\boldsymbol{B}}, \hat{\boldsymbol{T}})=\arg \min _{\boldsymbol{B}, \boldsymbol{T}}\|\boldsymbol{S}-\boldsymbol{B}-\boldsymbol{T}\|_{F}^{2}+\lambda\|\boldsymbol{B}\|_{1}+\|\boldsymbol{T}\|_{*}
$$

where $\|.\|_{F}^{2}$ is the Frobenus norm. $\lambda>0$ is a hyper-parameter to be tuned, balancing the trade-off between the sparsity of the blood and low-rankness of the tissues. To solve the convex optimization problem above, many algorithms exist in the literature, such as the one proposed in [4]. This method is referred to as robust principal component analysis (RPCA). A comprehensive review and analysis of this kind of methods can be found in [9]. Some of these techniques have been revisited in ultrasound imaging by Khoury et al. [10], by Bayat et al. [7] who solved this problem, assuming that $\boldsymbol{B}$ is sparse in the Fourier domain, or by Sathyanarayna et al. [8] who investigated the same problem via a sparse coding through expressing $\boldsymbol{B}$ in a specific dictionary.

One classical way for solving (2) is to use the Alternating Direction Method of Multipliers (ADMM) framework. ADMM aims at finding the solution of a complex optimization problem by accounting for easy-to-solve sub-problems. The minimization problem considered within ADMM is as follows:

$$
(\hat{\boldsymbol{x}}, \hat{\boldsymbol{z}})=\arg \min _{\hat{\boldsymbol{x}}, \hat{\boldsymbol{z}}} f(\boldsymbol{x})+g(\boldsymbol{z}) \quad \text { s.t. } \quad \boldsymbol{A} \boldsymbol{x}+\boldsymbol{B} \boldsymbol{z}=\boldsymbol{C}
$$


where $f(\boldsymbol{x})$ and $g(\boldsymbol{z})$ are convex functions and $\boldsymbol{A}, \boldsymbol{B}$ and $\boldsymbol{C}$ are matrices of appropriate sizes. The augmented Lagrangian associated to (3) is:

$$
\begin{aligned}
L(\boldsymbol{x}, \boldsymbol{z}, \boldsymbol{\nu}) & =f(\boldsymbol{x})+g(\boldsymbol{z})+\boldsymbol{\nu}^{T}(C-A \boldsymbol{x}-B \boldsymbol{z})+\frac{\mu}{2}\|C-A \boldsymbol{x}-B \boldsymbol{z}\|_{2}^{2} \\
& \approx f(\boldsymbol{x})+g(\boldsymbol{z})+\frac{\mu}{2}\left\|C-A \boldsymbol{x}-B \boldsymbol{z}+\frac{1}{\mu} \boldsymbol{\nu}\right\|_{2}^{2}
\end{aligned}
$$

The main idea of ADMM is to minimize the augmented Lagrangian above by iteratively processing the following three steps ( $k$ stands for the iteration number) until convergence:

Step 1: $\quad \hat{\boldsymbol{x}}^{(k+1)}=\arg \min _{\hat{\boldsymbol{x}}} L\left(\boldsymbol{x}, \boldsymbol{z}^{(k)}, \boldsymbol{\nu}^{(k)}\right)$;

Step 2: $\quad \hat{\boldsymbol{z}}^{(k+1)}=\arg \min _{\hat{\boldsymbol{z}}} L\left(\boldsymbol{x}^{(k+1)}, \boldsymbol{z}, \boldsymbol{\nu}^{k}\right)$;

Step 3: $\quad \boldsymbol{\nu}^{(k+1)}=\boldsymbol{\nu}^{(k)}+\mu\left(C-A \boldsymbol{x}^{(k+1)}-B \boldsymbol{z}^{(k+1)}\right)$.

Applying ADMM to (2) leads to the following augmented Lagrangian:

$$
L(\boldsymbol{B}, \boldsymbol{T}, \boldsymbol{\nu})=\lambda\|\boldsymbol{B}\|_{1}+\rho\|\boldsymbol{T}\|_{*}+\frac{\mu}{2}\left\|\boldsymbol{S}-\boldsymbol{B}-\boldsymbol{T}+\frac{1}{\mu} \boldsymbol{\nu}\right\|_{2}^{2}
$$

Finally, at each iteration, RPCA algorithm consists in the following three steps:

- Step 1:

$$
\hat{\boldsymbol{B}}^{(k+1)}=\arg \min _{\hat{\boldsymbol{B}}}\left(\lambda\|\boldsymbol{B}\|_{1}+\frac{\mu}{2}\left\|\boldsymbol{B}-\left(\boldsymbol{S}-\boldsymbol{T}^{(k)}+\frac{1}{\mu} \boldsymbol{\nu}^{(k)}\right)\right\|_{2}^{2}\right) ;
$$

- Step 2:

$\hat{\boldsymbol{T}}^{(k+1)}=\arg \min _{\hat{\boldsymbol{T}}}\left(\|\boldsymbol{T}\|_{*}+\frac{\mu}{2}\left\|\boldsymbol{T}-\left(\boldsymbol{S}-\boldsymbol{B}^{(k+1)}+\frac{1}{\mu} \boldsymbol{\nu}^{(k)}\right)\right\|_{2}^{2}\right) ;$

- Step 3:

$$
\boldsymbol{\nu}^{(k+1)}=\boldsymbol{\nu}+\mu\left(\boldsymbol{S}-\boldsymbol{B}^{(k+1)}-\boldsymbol{T}^{(k+1)}\right) .
$$

Note that steps 1 and 2 above are both convex problems having closed-form solutions: soft thresholding [11] for step 1 , and singular value thresholding (SVT) [12] for step 2.

Despite its efficiency, the previous algorithm does not explicitly take into account the limited resolution of Doppler data caused by the system impulse response (i.e., the PSF).

\section{A. Proposed method: joint deconvolution and RPCA}

In order to estimate a high-resolution blood flow, we propose to deconvolve $\boldsymbol{B}$, using a measured system impulse response (PSF). Indeed, $\boldsymbol{B}$ and $\boldsymbol{T}$ have limited spatial resolution partly because of the PSF. Specifically, we consider that $\boldsymbol{B}$ and $T$ can be expressed as:

$$
\begin{aligned}
& \boldsymbol{B}=H \boldsymbol{x} \\
& \boldsymbol{T}=H \boldsymbol{y}
\end{aligned}
$$

where $H \in \mathbb{C}^{N_{z} N_{x} N_{t} \times N_{z} N_{x} N_{t}}$ stands for the system PSF, $\boldsymbol{x} \in \mathbb{C}^{N_{z} N_{x} \times N_{t}}$ and $\boldsymbol{y} \in \mathbb{C}^{N_{z} N_{x} \times N_{t}}$ are respectively the high-resolution blood and tissue to be estimated. Note that for computational efficiency, we assume circular convolution with periodic boundary extensions, that allows $H$ to be a block circulant with circulant blocks (BCCB) matrix diagonalisable in the Fourier domain (see, e.g., [13]). In this work, we are only interested in the blood term $\boldsymbol{x}$, and we thus continue using the low-resolution tissue component $\boldsymbol{T}$ in the following developments instead of its high-resolution counterpart $\boldsymbol{y}$. Finally, to separate $\boldsymbol{x}$ and $\boldsymbol{T}$ from data $\boldsymbol{S}$, we propose a joint
Deconvolution and RPCA formulated as an objective function to minimize as follows:

$$
(\hat{\boldsymbol{x}}, \hat{\boldsymbol{T}})=\underset{\hat{\boldsymbol{x}}, \hat{\boldsymbol{T}}}{\arg \min _{\mathrm{T}}}\|\boldsymbol{S}-H \boldsymbol{x}-\boldsymbol{T}\|_{F}^{2}+\lambda\|\boldsymbol{x}\|_{1}+\|\boldsymbol{T}\|_{*}
$$

Similar to RPCA, (7) can be also solved with ADMM, for instance by using an auxiliary variable $\boldsymbol{z}$ [14]. This results in:

$$
(\hat{\boldsymbol{x}}, \hat{\boldsymbol{z}}, \hat{\boldsymbol{T}})=\arg \min _{\hat{\boldsymbol{x}}, \hat{\boldsymbol{z}}, \hat{\boldsymbol{T}}}\|\boldsymbol{S}-H \boldsymbol{x}-\boldsymbol{T}\|_{F}^{2}+\|\boldsymbol{x}-\boldsymbol{z}\|_{F}^{2}+\lambda\|\boldsymbol{x}\|_{1}+\|\boldsymbol{T}\|_{*}
$$

The detailed optimization process of minimizing (8), named joint Deconvolution and RPCA (DRPCA) in the following, is resumed in Algorithm 1.

\section{Algorithm 1 Joint deconvolution and RPCA \\ Input: observations $\boldsymbol{S}$ and PSF $\boldsymbol{H}$; hyper-parameters $\lambda, \mu$ and tolerance.}

Output: high-resolution blood $x$ and tissue $T$.

Initialize: $\boldsymbol{x}=\boldsymbol{z}=0, \boldsymbol{T}=0, \boldsymbol{\nu}=0, \boldsymbol{w}=0, \mu_{\min }=10^{-6}, \quad \epsilon=2$, $\mu_{\max }=10^{6}$, and tolerance $=10^{-6}$;

while not converged do

1) update $\boldsymbol{T}$ and keep other variables by

$$
\begin{aligned}
\boldsymbol{T}= & \arg \min _{\hat{\boldsymbol{T}}}\left(\|\boldsymbol{T}\|_{*}+\frac{\mu}{2}\left\|\boldsymbol{T}-\left(\boldsymbol{S}-\boldsymbol{H} \boldsymbol{x}+\frac{1}{\mu} \boldsymbol{\nu}\right)\right\|_{F}^{2}\right) \\
= & \text { Soft }_{\frac{\rho}{\mu}}[\triangle] V^{T} \\
& \text { with } \quad \boldsymbol{S}-\boldsymbol{H} \boldsymbol{z}^{k}+\frac{1}{\mu} \boldsymbol{\nu}^{k}=U \triangle V^{T}
\end{aligned}
$$

2) update $z$ and keep other variables by

$$
\begin{aligned}
\boldsymbol{z} & =\arg \min _{\hat{\boldsymbol{z}}}\left(\lambda\|\boldsymbol{z}\|_{1}+\frac{\mu}{2}\left\|\boldsymbol{z}-\left(\boldsymbol{x}+\frac{1}{\mu} \boldsymbol{w}\right)\right\|_{F}^{2}\right. \\
& =\text { Soft }_{\frac{\lambda}{\mu}}\left[\boldsymbol{x}^{k}+\frac{1}{\mu} \boldsymbol{w}^{k}\right]
\end{aligned}
$$

3) update $\boldsymbol{x}$ and keep other variables by

$$
\begin{aligned}
\boldsymbol{x}^{k+1} & =\arg \min _{\boldsymbol{x}}\left(\frac{\mu}{2}\left\|\boldsymbol{S}-\boldsymbol{H} \boldsymbol{x}-\boldsymbol{T}^{k+1}+\frac{1}{\mu} \boldsymbol{\nu}^{k}\right\|_{F}^{2}\right. \\
& \left.+\frac{\mu}{2}\left\|\boldsymbol{x}-\boldsymbol{z}^{k+1}+\frac{1}{\mu} \boldsymbol{w}^{k}\right\|_{F}^{2}\right) \\
& =\boldsymbol{F}\left(\boldsymbol{D}^{T} \boldsymbol{D}+\boldsymbol{I}\right)^{-1} \boldsymbol{F}^{T}\left[\boldsymbol{F} \boldsymbol{D}^{T} \boldsymbol{F}^{T}\left(\boldsymbol{S}-\boldsymbol{T}^{\boldsymbol{k}+\mathbf{1}}\right)\right. \\
& \left.+\boldsymbol{z}^{k+1}+\frac{1}{\mu}\left(\boldsymbol{F} \boldsymbol{D}^{T} \boldsymbol{F}^{T} \boldsymbol{\nu}^{k}-\boldsymbol{w}^{k}\right)\right]
\end{aligned}
$$

4) update the multipliers by

$$
\begin{aligned}
\boldsymbol{\nu}^{k+1} & =\boldsymbol{\nu}^{k}+\mu(\boldsymbol{S}-\boldsymbol{H} \boldsymbol{x}-\boldsymbol{T}) \\
\boldsymbol{w}^{k+1} & =\boldsymbol{w}^{k}+\mu(\boldsymbol{x}-\boldsymbol{z})
\end{aligned}
$$

5) update the parameter $\mu$ by $\mu=\min \left(\epsilon \mu, \mu_{\max }\right)$

6) check the convergence conditions:

$\left(\|\boldsymbol{S}-\boldsymbol{H} \boldsymbol{x}-\boldsymbol{T}\|_{F} /\|\boldsymbol{S}\|_{F}\right)<$ tolerance

$\left(\|\boldsymbol{x}-\boldsymbol{z}\|_{F} /\|\boldsymbol{S}\|_{F}\right)<$ tolerance

end

\section{RESULTS}

To evaluate the performance of the proposed method, we applied it to simulated, in vitro and in vivo data. 


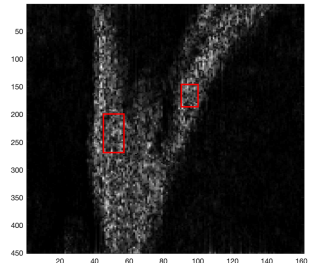

(a) Simulated data before convo- (b) Simulated data after convolulution

Fig. 1. B-mode images corresponding to simulated flow data.

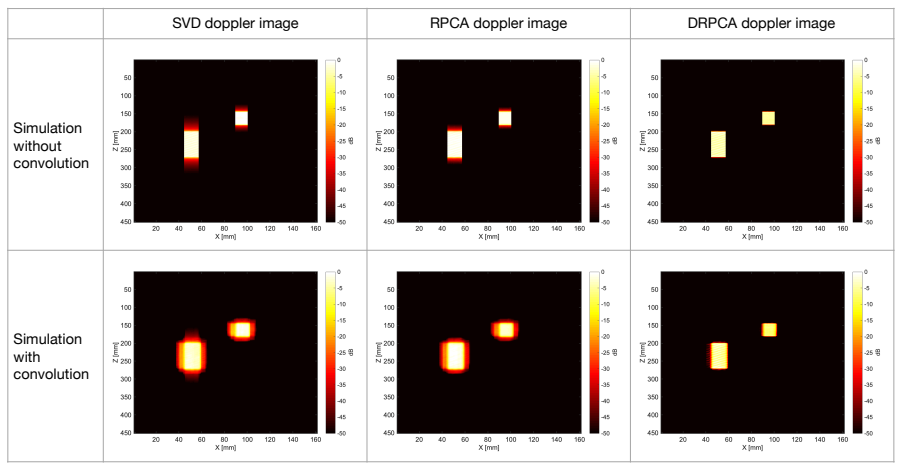

Fig. 2. Power Doppler images from simulated data estimated by respectively SVD, RPCA and DRPCA.

\section{A. Simulation results}

The simulated data, consisting in randomly distributed scatterers with Gaussian random amplitudes, represents a static vessel that plays the role of static tissue. Two moving rectangles were also simulated inside the vessel mimicking the blood flow. Furthermore, the generated reflectivity maps were convolved with an ultrasound PSF measured experimentally in vitro. Fig. 1 depicts the resulting B-mode images before (a) and after (b) convolution with the PSF.

Fig. 2 depicts the power Doppler estimation results of blood flow obtained on simulated data using SVD, RPCA and the proposed DRPCA. In particular, the upper line in Fig. 2 corresponds to the results from data before convolution, while the lower line shows the same results but from data after convolution with the PSF. One may observe that power Doppler images with data including the PSF are generally more blurred than those obtained from native data. Compared to SVD and RPCA, both power Doppler images estimated with DRPCA present sharper edges by efficiently removing the blur around the two rectangles. This proves the abbility of the proposed approach to estimate high-resolution and highsensitivity flow. Note that the thresholds used within SVD were manually tuned to their best values.

\section{B. In vitro results}

In vitro experiments consisted of a flow inside a small tube with a laminar flow (blood mimicking liquid). The acquisition was performed with a programmable platform (Verasonics Vantage) equipped with an L11-4v probe. Ultrafast imaging

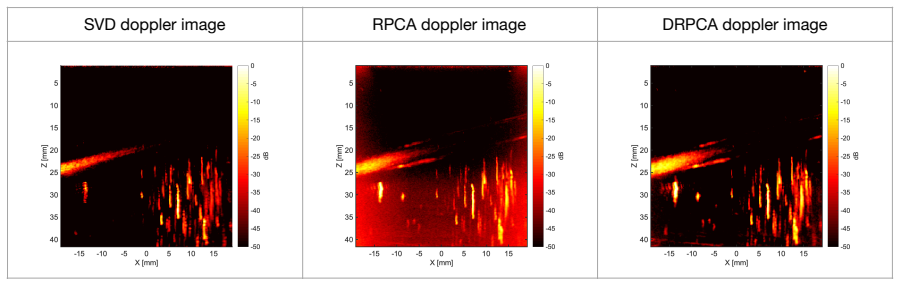

Fig. 3. Power doppler images from in vitro data estimated by respectively SVD, RPCA and DRPCA.

was performed with tilted plane wave emissions using 11 angles $\left[-5^{\circ}: 1^{\circ}: 5^{\circ}\right]$ emitted at a PRF of $14 \mathrm{kHz}$, in order to achieve a frame rate of $1.28 \mathrm{kHz}$ after coherent compounding. This set of angles was repeated 400 times to build ultrafast cineloop during 312 msec. SVD decomposition was performed in parallel for performance comparison. The PSF was measured experimentally in vitro with the same acquisition sequence.

The results of power Doppler on in vitro data are displayed in Fig. 3, highlighting the differences between SVD, RPCA and DRPCA. One may observe that RPCA is noisier than SVD and DRPCA while DRPCA provides better spatial resolution and higher sensitivity blood flow estimation.

\section{In vivo data results}

The proposed DRPCA approach was also evaluated on data acquired from a healthy brain region. The ultrasound data was acquired during an open skull operation at the neurosurgery department of CHRU Tours. Fig. 4 visually demonstrates that power Doppler images estimated with SVD filtering and RPCA are both noisier than DRPCA. Moreover, one may also observe the finer degree of details of brain vascularization enabled by DRPCA.

\section{Hyperparameter tunning}

In this subsection, we explain how the two hyper-parameters $\lambda$ and $\mu$ used with RPCA and DRPCA approaches were tunned in this work. Parameter $\lambda$ : Both with RPCA and DRPCA, $\lambda=\frac{1}{\sqrt{\max (N z * N x, N t)}}$ was set as suggested in [4]. Furthermore, $\lambda$ is refined to get the best Doppler image. When the value of $\lambda$ is manually decreased, noise and blood are better preserved for both RPCA and DRPCA methods. Indeed, $\lambda$ weights the sparse component of the signal. It tends to reduce the sparsity of the result while decreasing, consequently turning the blood flow brighter. Parameter $\mu$ : With RPCA, $\mu$ was chosen as $\mu=10 \times \lambda$ as proposed in [4] while with DRPCA, $\mu$ was multiplied by a fixed value, (herein by 2) and updated iteratively. Parameter $\mu$ has an impact on the convergence speed of the algorithm. When it is reduced, convergence accelerates. A reduction in noise is observed when the value of the convergence parameter is decreased.

\section{Discussion}

Three methods for blood and tissue subspace separation were compared in this work. The three methods presented pre- 


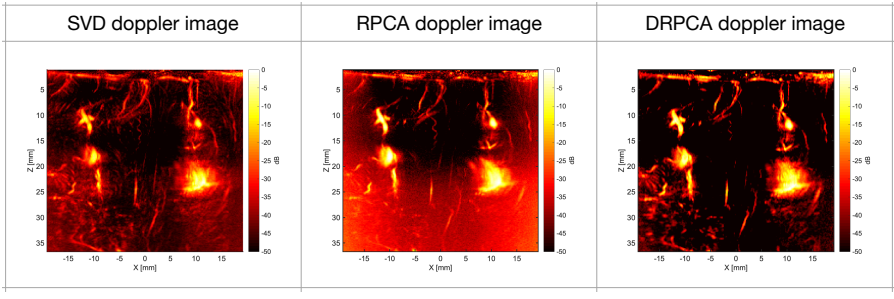

Fig. 4. The power doppler images of brain by respectively SVD, RPCA, DRPCA

viously, SVD, RPCA and DRPCA, have their own advantages and disadvantages.

SVD is a straightforward and easy-to-implement method, based on the correlation of spatial singular vectors magnitude. However, it requires a manual tuning of the two thresholds needed to separate tissue, blood and noise components, to visualize only the blood flow. From this perspective, SVD may not be reproducible from one operator to another. The selection of blood directly depends on the selection of these thresholds, without any further control on the result.

RPCA algorithm is more complex than classical SVD, It estimates the two main components, tissue and blood, by exploiting their low-rank or sparse properties, leading to an automatic method without the need of manually tuning the parameters. Therefore, for the same sequences, RPCA yields the same result if there is no change in the parameters $\lambda$ and $\mu$. Moreover, it uses parameters on which one can play to optimize the result. This method is therefore more advantageous and easier to handle.

DRPCA algorithm is based upon RPCA, sharing roughly all its advantages and disadvantages. However, it provides a better resolution for the observed blood flow due to its deconvolution ability and is able to better decrease the level of noise in the results. Its most important drawback is the computational cost related to deconvolution.

\section{Conclusion}

In this paper, we proposed an estimation method for separating the blood flow using ultrafast sequences. Our method, based on ADMM optimization, performs joint deconvolution and RPCA using the measured system PSF leading to high resolution and high sensitivity flows, at the cost of high computational cost. Our future focus will be the automatic adjustment of hyper-parameters and the reduce of computational load.

\section{REFERENCES}

[1] C. Demené, T. Deffieux, M. Pernot, B. F. Osmanski, V. Biran, J. L. Gennisson, L. A. Sieu, A. Bergel, S. Franqui, J. M. Correas, I. Cohen, O. Baud, and M. Tanter, "Spatiotemporal clutter filtering of ultrafast ultrasound data highly increases doppler and fultrasound sensitivity," IEEE Transactions on Medical Imaging, vol. 34, no. 11, pp. 2271-2285, Nov. 2015.

[2] J. Baranger, B. Arnal, F. Perren, O. Baud, M. Tanter, and C. Demené, "Adaptative spatiotemporal svd clutter filtering for ultrafast doppler imaging using similarity of spatial singular vectors," IEEE Transactions on Medical Imaging, vol. 37, no. 7, pp. 1574-1586, July 2018.
[3] R. A. Maronna, "Robust m-estimators of multivariate location and scatter," Ann. Stat., vol. 4, no. 1, pp. 51-67, 1976.

[4] J. Wright, A. Ganesh, S. Rao, Y. Peng, and Y. Ma, "Robust principal component analysis: Exact recovery of corrupted low-rank matrices via convex optimization," Proc. Neural Inf. Process. Syst, pp. 1-9, 2009.

[5] F. Torre and M. Black, "Robust principal component analysis for computer vision," Proc. Int. Conf. Comput. Vis., pp. 362-369, 2001.

[6] S. J. Devlin, R. Gnanadesikan, and J. R. Kettenring, "Robust estimation of dispersion matrices and principal components," J. Amer. Stat. Assoc., vol. 76, no. 374, pp. 354-362, 1981.

[7] M. Bayat and M. Fatemi, "Concurrent clutter and noise suppression via low rank plus sparse optimization for non-contrast ultrasound flow doppler processing in microvasculature," in IEEE International Conference on Acoustics, Speech and Signal Processing ICASSP'2018, Calgary, Canada, 2018.

[8] S. G. Sathyanarayna, S. T. Acton, and J. A. Hossack, "Suppression of clutter by rank adaptive reweighted sparse coding," in IEEE International Ultrasonics Symposium IUS'2017, Washington DC, USA, 2017.

[9] Thierry Bouwmans, Andrews Sobral, Sajid Javed, Soon Ki Jung, and El-hadi Zahzah, "Decomposition into low-rank plus additive matrices for background/foreground separation: A review for a comparative evaluation with a large-scale dataset," Computer Science Review, vol. 23.

[10] C.Barthelemy, E.Khoury, S.Beuve, I.Zemmoura, JL.Gennisson, A.Basarab, D.Kouamé, and JP.Reménieras, "Development of ultrasensitive doppler imaging method for the surgical management of open-brain tumors," in IEEE International Ultrasonics Symposium IUS'2019, Glasgow, UK, Oct. 2019.

[11] Z. Lin, M. Chen, L. Wu, and Y. Ma, "The augmented lagrange multiplier method for exact recovery of corrupted low-rank matrices," Dept. Electr. Comput. Eng., UIUC, Urbana, Tech. Rep., 2009.

[12] J. Cai, E. J. Candès, and Z. Shen, "A singular value thresholding algorithm for matrix completion," Soc. Ind. Appl. Math. J. Optim., vol. 20, no. 4, pp. 1956-1982, 2010.

[13] P. C. Hansen, J. G. Nagy, and D. P. O'Leary, Deblurring Images : Matrices, Spectra, and Filtering, Society for Industrial and Applied Mathematic, 2006.

[14] B. Bao, G. Liu, C. Xu, and S. Yan, "Inductive robust principal component analysis," IEEE transactions on image processing, vol. 21, no. 8, pp. 3794-3800, Aug. 2012. 